\title{
Unified Treatment of a Class of Spherically Symmetric Potentials: Quasi-Exact Solution
}

\author{
H. Panahi and M. Baradaran \\ Department of Physics, University of Guilan, Rasht 41635-1914, Iran \\ Correspondence should be addressed to H. Panahi; t-panahi@guilan.ac.ir
}

Received 12 May 2016; Accepted 19 June 2016

Academic Editor: Shi-Hai Dong

Copyright @ 2016 H. Panahi and M. Baradaran. This is an open access article distributed under the Creative Commons Attribution License, which permits unrestricted use, distribution, and reproduction in any medium, provided the original work is properly cited. The publication of this article was funded by $\mathrm{SCOAP}^{3}$.

\begin{abstract}
We investigate the Schrödinger equation for a class of spherically symmetric potentials in a simple and unified manner using the Lie algebraic approach within the framework of quasi-exact solvability. We illustrate that all models give rise to the same basic differential equation, which is expressible as an element of the universal enveloping algebra of $s(2)$. Then, we obtain the general exact solutions of the problem by employing the representation theory of sl(2) Lie algebra.
\end{abstract}

\section{Introduction}

From the viewpoint of solvability, the spectral problems are divided into two main classes: the exactly solvable (ES) models and exactly nonsolvable models. A quantum model is called ES if, for all its energy levels and corresponding wavefunctions, explicit expressions can be determined algebraically. These models are distinguished by the fact that there is a natural basis in the Hilbert space in which the infinitedimensional Hamiltonian can be diagonalized with the help of algebraic methods. In the literature, considerable efforts have been devoted to obtaining the exact solutions of the relativistic and nonrelativistic equations using different methods and techniques [1-5]. In contrast, the exactly nonsolvable models are the spectral problems whose infinite-dimensional Hamiltonian cannot be diagonalized algebraically. Unfortunately, there are only a small number of potentials for which the Schrödinger equation can be solved exactly, like the harmonic oscillator [6, 7], Pöschl-Teller [8, 9], Coulomb [10-12], Morse [13, 14], Rosen-Morse [15], Manning-Rosen [16, 17], Tietz [18], and so forth [19-22]. In 1980s, the series of papers by Shifman, Ushveridze, and Turbiner was devoted to the introduction of an intermediate class between the ES and the exactly nonsolvable models for which a certain finite number of eigenvalues and eigenfunctions, but not the whole spectrum, can be calculated exactly by algebraic methods.
They were called quasi-exactly solvable (QES) [23-26]. These models are distinguished by the fact that the Hamiltonian is expressible as a quadratic combination of the generators of a finite-dimensional Lie algebra of first-order differential operators preserving a finite-dimensional subspace of functions and thereby can be represented as a block-diagonal matrix with at least one finite block. Thus, the problem reduces to diagonalizing this block and computing the corresponding eigenvalues and eigenfunctions, which always can be done. In this paper, using the Lie algebraic approach, we present a simple unified derivation and exact solution of the Schrödinger equation for a class of four spherically symmetric potentials within the framework of quasi-exact solvability. We demonstrate that all four cases are reducible to the same basic differential equation which can be solved exactly due to the existence of a hidden sl(2) symmetry. Then, with the aid of the representation theory of $\mathrm{sl}(2)$, the general exact solution to the basic equation is determined.

This paper is organized as follows: in Section 2, we briefly review the Lie algebraic approach of quasi-exact solvability. In Section 3, we introduce the four systems and transform the corresponding equations into the same form that is suitable for sl(2) algebraization and can be expressed as an element of the universal enveloping algebra of $\mathrm{sl}(2)$. Then, using the representation theory of $\mathrm{sl}(2)$, we obtain the general exact 
solution to the basic equation in Section 4. Also, the closedform expressions for the eigenvalues and eigenfunctions as well as the allowed parameters of potentials are given for each of the systems. We end with conclusions in Section 5.

\section{Quasi-Exact Solvability through the sl(2) Algebraization}

The general problem of quantum mechanics is to solve the Schrödinger equation $H \Psi=E \Psi$, where the wavefunction $\Psi$ belongs to the space of square integrable functions $L^{2}(R)$. An eigenvalue equation is called QES if there is a nontrivial finitedimensional subspace $U$ of $L^{2}(R)$ which is invariant under $H$ : that is, $H U \subseteq U[25,26]$. According to [25], any onedimensional QES differential equation possesses a hidden Lie algebra sl(2) which is the only Lie algebra of first-order differential operators that possesses a finite-dimensional representation. This implies that they can be rewritten in terms of $\mathrm{sl}(2)$ generators with differential operators [23-25]:

$$
\begin{aligned}
& J_{n}^{+}=-x^{2} \frac{d}{d x}+n x, \\
& J_{n}^{0}=x \frac{d}{d x}-\frac{n}{2}, \\
& J_{n}^{-}=\frac{d}{d x},
\end{aligned}
$$

which obey the commutation relations

$$
\begin{aligned}
& {\left[J_{n}^{+}, J_{n}^{-}\right]=2 J_{n}^{0},} \\
& {\left[J_{n}^{0}, J_{n}^{ \pm}\right]= \pm J_{n}^{ \pm},}
\end{aligned}
$$

and leave invariant the $(n+1)$-dimensional vector space of polynomials:

$$
P_{n+1}(x)=\left\langle 1, x, x^{2}, \ldots, x^{n}\right\rangle .
$$

With these properties, it is easy to verify that the most general second-order differential operator in the enveloping algebra of $\mathrm{sl}(2)$ takes the form

$$
H=\sum_{a, b=0, \pm} C_{a b} J^{a} J^{b}+\sum_{a=0, \pm} C_{a} J^{a}+C,
$$

where $C_{a b}, C_{a}$, and $C$ are real constants. On the other hand, this operator as an ordinary differential equation has the general form

$$
\begin{aligned}
H \phi(x) & =0, \\
H & =-P_{4}(x) \frac{d^{2}}{d x^{2}}+P_{3}(x) \frac{d}{d x}+P_{2}(x),
\end{aligned}
$$

where $P_{j}$ are the polynomials of degree $j$. This operator can be turned into the Schrödinger-like operator:

$$
\begin{aligned}
\widetilde{H} & =e^{-A(z)} H e^{A(z)} \\
& =-\frac{1}{2} \frac{d^{2}}{d z^{2}}+\left(A^{\prime}\right)^{2}-A^{\prime \prime}+P_{2}(x(z)),
\end{aligned}
$$

through the following change of variable and gauge transformation:

$$
\begin{gathered}
z= \pm \int \frac{d x}{\sqrt{P_{4}}}, \\
\phi(x)=e^{-\int\left(P_{3} / P_{4}\right) d x+\log z^{\prime}} \psi(z) .
\end{gathered}
$$

In the next section, using the method given above, we show that exact solutions of the Schrödinger equation for the four models can be simply obtained in a unified treatment.

\section{The Four Models and the Corresponding Differential Equations}

In this section, we introduce the models which will be the object of study in this paper. For each case, we illustrate that the corresponding Schrödinger equation is reducible to the basic differential equation of second order which is QES due to the existence of a hidden sl(2) algebraic structure.

3.1. Nonpolynomial Potential. First, we consider the nonpolynomial oscillator defined as [27]

$$
V(r)=r^{2}+\frac{\alpha r^{2}}{1+\beta r^{2}},
$$

where $\beta>0$ and $\alpha$ is a real constant. This potential appears in various branches of physics such as the zero-dimensional quantum field theory with nonlinear Lagrangian [28, 29], quantum mechanics [30, 31], laser physics [32, 33], and so forth. This potential has been studied by a variety of methods including the analytic continued fractions [34], the supersymmetric quantum mechanics [35], the $1 / N$ expansion method [27], the wavefunction ansatz method [12], and the Bethe ansatz method [36]. In atomic units $(m=\hbar=c=1)$, the radial Schrödinger equation with potential (8) is

$$
\left(-\frac{1}{2} \frac{d^{2}}{d r^{2}}+\frac{l(l+1)}{2 r^{2}}+r^{2}+\frac{\alpha r^{2}}{1+\beta r^{2}}-E\right) \psi(r)=0 .
$$

Using the change of variable $z=-\beta r^{2}$ and making the gauge transformation

$$
\psi(z)=(1-z) z^{(l+1) / 2} e^{-z / \beta \sqrt{2}} \varphi(z),
$$

which preserves the asymptotic behaviour of the wavefunction at the origin and infinity, (9) becomes

$$
\begin{aligned}
& \left\{z(z-1) \frac{d^{2}}{d z^{2}}\right. \\
& \quad+\left(-\frac{2}{\beta \sqrt{2}} z^{2}+\left(\frac{2}{\beta \sqrt{2}}+l+\frac{7}{2}\right) z-\left(l+\frac{3}{2}\right)\right) \frac{d}{d z} \\
& \quad+\left(-\frac{E}{2 \beta}+\frac{\alpha}{2 \beta^{2}}-\frac{1}{\beta \sqrt{2}}\left(l+\frac{7}{2}\right)\right) z+\left(\frac{E}{2 \beta}+\frac{l}{2}\right. \\
& \left.\left.\quad+1+\frac{1}{\beta \sqrt{2}}\left(l+\frac{3}{2}\right)\right)\right\} \varphi(z)=0 .
\end{aligned}
$$


3.2. Screened Coulomb Potential. Here, we consider the screened Coulomb potential defined by [37]

$$
V(r)=\frac{\gamma}{r}+\frac{\delta}{r+\kappa}, \quad \gamma<-\delta
$$

The corresponding radial Schrödinger equation is given by

$$
\left(-\frac{1}{2} \frac{d^{2}}{d r^{2}}+\frac{l(l+1)}{2 r^{2}}+\frac{\gamma}{r}+\frac{\delta}{r+\kappa}\right) \psi(r)=E \psi(r) .
$$

Several methods and techniques for solving this problem can be found in [12, 36-39]. Applying the transformation

$$
\psi(r)=(r+\kappa) r^{l+1} e^{-\sqrt{-2 E}(r+\kappa)} \varphi(r)
$$

and also replacing the variable $r$ by $z$, we obtain

$$
\begin{aligned}
& \left\{z(z+\kappa) \frac{d^{2}}{d z^{2}}+2\left(-\sqrt{-2 E} z^{2}+(-\sqrt{-2 E} \kappa+l+2) z\right.\right. \\
& +(l+2) \kappa) \frac{d}{d z}+(2(-\delta-\gamma-\sqrt{-2 E}(l+2)) z \\
& \quad-2(\kappa(l+1) \sqrt{-2 E}+\kappa \gamma-l-1))\} \varphi(z)=0 .
\end{aligned}
$$

3.3. Singular Integer Power Potential. The problem of the singular power potentials has been widely carried out in various branches of physics, in both classical and quantum mechanics [40-43]. Here, we consider the singular integer power potential as $[44,45]$

$$
V(r)=\frac{\lambda}{r}+\frac{\mu}{r^{2}}+\frac{\xi}{r^{3}}+\frac{\tau}{r^{4}}
$$

with the corresponding Schrödinger equation given by

$$
\begin{aligned}
& \left(-\frac{1}{2} \frac{d^{2}}{d r^{2}}+\frac{l(l+1)}{2 r^{2}}+\frac{\lambda}{r}+\frac{\mu}{r^{2}}+\frac{\xi}{r^{3}}+\frac{\tau}{r^{4}}\right) \psi(r) \\
& =E \psi(r) .
\end{aligned}
$$

From the asymptotic behaviour of the wavefunction, we consider the following transformation:

$$
\psi(r)=r^{1+\xi / \sqrt{2 \tau}} e^{-(\sqrt{-2 E} r+\sqrt{2 \tau} / r)} \varphi(r) .
$$

Substituting this into (17) and also replacing $r$ by $z$, we get

$$
\begin{aligned}
& \left\{z^{2} \frac{d^{2}}{d z^{2}}+2\left(-\sqrt{-2 E} z^{2}+\left(1+\frac{\xi}{\sqrt{2 \tau}}\right) z+\sqrt{2 \tau}\right) \frac{d}{d z}\right. \\
& -2\left(\sqrt{-2 E}\left(1+\frac{\xi}{\sqrt{2 \tau}}\right)+\lambda\right) z-2 \mu-l(l+1) \\
& \left.-2 \sqrt{-4 \tau E}+\frac{\xi}{\sqrt{2 \tau}}+\frac{\xi^{2}}{2 \tau}\right\} \varphi(z)=0 .
\end{aligned}
$$

3.4. Singular Anharmonic Potential. Here, following [46], we consider the potential

$$
V(r)=\omega r^{2}+\frac{\varepsilon}{r^{2}}+\frac{\sigma}{r^{4}}+\frac{\chi}{r^{6}}
$$

with the radial Schrödinger equation:

$$
\begin{aligned}
& \left(-\frac{1}{2} \frac{d^{2}}{d r^{2}}+\frac{l(l+1)}{2 r^{2}}+\omega r^{2}+\frac{\varepsilon}{r^{2}}+\frac{\sigma}{r^{4}}+\frac{\chi}{r^{6}}\right) \psi(r) \\
& =E \psi(r) .
\end{aligned}
$$

Similar to the previous cases, we extract the asymptotic behaviour of the wavefunction by making the following transformations:

$$
\begin{gathered}
z=r^{2}, \\
\psi(r)=r^{3 / 2+\sigma / \sqrt{2 \chi}} e^{-\left(\sqrt{\omega / 2} r^{2}+\sqrt{\chi / 2}\left(1 / r^{2}\right)\right)} \varphi(r) .
\end{gathered}
$$

After substituting this into (21), we obtain

$$
\begin{aligned}
& \left\{z^{2} \frac{d^{2}}{d z^{2}}+\left(-\sqrt{2 \omega} z^{2}+\left(2+\frac{\sigma}{\sqrt{2 \chi}}\right) z+\sqrt{2 \chi}\right) \frac{d}{d z}\right. \\
& +\left(\frac{E}{2}-\sqrt{\frac{\omega}{2}}\left(2+\frac{\sigma}{\sqrt{2 \chi}}\right)\right) z \\
& \left.-\frac{1}{4}\left(l^{\prime}\left(l^{\prime}+1\right)+2 \sqrt{4 \omega \chi}-\frac{\sigma^{2}}{2 \chi}-\frac{2 \sigma}{\sqrt{2 \chi}}-\frac{3}{4}\right)\right\} \\
& \cdot \varphi(z)=0,
\end{aligned}
$$

where

$$
l^{\prime}=\frac{-1+\sqrt{4 l^{2}+4 l+8 \varepsilon+1}}{2} .
$$

\section{Solutions of the Basic Differential Equation for the Four Models}

In the previous section, we have shown that our QES models, after the appropriate transformations, are expressible as second-order differential equations (11), (15), (19), and (23), respectively. These equations have the same basic structure:

$$
\begin{aligned}
H \phi(z)= & 0, \\
H= & z(z-a) \frac{d^{2}}{d z^{2}}+\left(b_{2} z^{2}+b_{1} z+b_{0}\right) \frac{d}{d z} \\
& +\left(c_{1} z+c_{0}\right),
\end{aligned}
$$

where $a, c_{0}, c_{1}, b_{0}, b_{1}$, and $b_{2}$ are real constants. Here, we intend to solve this equation using the Lie algebraic approach within the representation theory of $\mathrm{sl}(2)$. More precisely, from (4), the general form of a one-dimensional QES differential equation is as follows [25]: 


$$
\begin{aligned}
H= & C_{++} J_{n}^{+} J_{n}^{+}+C_{+0} J_{n}^{+} J_{n}^{0}+C_{+-} J_{n}^{+} J_{n}^{-}+C_{0-} J_{n}^{0} J_{n}^{-} \\
& +C_{--} J_{n}^{-} J_{n}^{-}+C_{+} J_{n}^{+}+C_{0} J_{n}^{0}+C_{-} J_{n}^{-}+C
\end{aligned}
$$

which clearly preserves the $(n+1)$-dimensional representation space of the $\mathrm{sl}(2)$ algebra as

$$
\phi_{n}(z)=\sum_{m=0}^{n} p_{m} z^{m}, \quad n=0,1,2, \ldots
$$

Comparing (25) with (26), it is seen that the differential operator $H$ can be expressed as a special case of the general form (26) as

$$
\begin{aligned}
H= & -J_{n}^{+} J_{n}^{-}-a J_{n}^{0} J_{n}^{-}-b_{2} J_{n}^{+}+\left(n+b_{1}\right) J_{n}^{0} \\
& +\left(b_{0}-\frac{n}{2} a\right) J_{n}^{-}+\left(\frac{n^{2}}{2}+\frac{n}{2} b_{1}+c_{0}\right),
\end{aligned}
$$

if the following constraint on the coefficients holds:

$$
c_{1}=-n b_{2}
$$

Hence, we have shown that the differential operator $H$ is an element of the universal enveloping algebra of $\mathrm{sl}(2)$ and thereby we can use the representation theory of $\mathrm{sl}(2)$ to determine the solutions of the problem. In the $(n+1)$ dimensional space $\phi_{n}(z)$, the operators $J_{n}^{+}, J_{n}^{0}$, and $J_{n}^{-}$can be represented by the $(n+1) \times(n+1)$ matrices in the basis $\left\langle 1, z, z^{2}, \ldots, z^{n+1}\right\rangle$ :

$$
J_{n}^{+}=\left(\begin{array}{cccccc}
0 & 1 & 0 & \cdots & 0 & 0 \\
0 & 0 & 2 & \cdots & 0 & 0 \\
\vdots & \vdots & \cdots & 3 & \vdots & \vdots \\
\vdots & \vdots & \cdots & \cdots & n-1 & 0 \\
0 & 0 & \cdots & \cdots & 0 & n \\
0 & 0 & \cdots & \cdots & 0 & 0
\end{array}\right)
$$$$
J_{n}^{-}=\left(\begin{array}{cccccc}
0 & 0 & 0 & \cdots & 0 & 0 \\
n & 0 & 0 & \cdots & 0 & 0 \\
\vdots & n-1 & \cdots & \cdots & \vdots & \vdots \\
\vdots & \vdots & \cdots & \cdots & 0 & 0 \\
0 & 0 & \cdots & 2 & 0 & 0 \\
0 & 0 & \cdots & \cdots & 1 & 0
\end{array}\right)
$$

$$
J_{n}^{0}=\left(\begin{array}{cccccc}
\frac{n}{2} & 0 & \cdots & \cdots & 0 & 0 \\
0 & \frac{n}{2}-1 & \cdots & \cdots & 0 & 0 \\
\vdots & \vdots & \cdots & \cdots & \vdots & \vdots \\
0 & 0 & \cdots & \cdots & 1-\frac{n}{2} & 0 \\
0 & 0 & \cdots & \cdots & 0 & -\frac{n}{2}
\end{array}\right) .
$$

Substituting (30) into (28) results in a matrix equation whose nontrivial solution exists if the following condition is fulfilled (Cramer's rule):

$$
\left|\begin{array}{ccccc}
c_{0} & b_{0} & 0 & 0 & 0 \\
-n b_{2} & c_{0}+b_{1} & 2 b_{0}-2 a & 0 & 0 \\
0 & -(n-1) b_{2} & c_{0}+2 b_{1}+2 & \ddots & 0 \\
0 & \ddots & \ddots & \ddots & \vdots \\
0 & 0 & -2 b_{2} & c_{0}+(n-1)\left(b_{1}+n-2\right) & n\left(b_{0}-(n-1) a\right) \\
0 & 0 & 0 & -b_{2} & c_{0}+n\left(b_{1}+n-1\right)
\end{array}\right|=0
$$

This equation yields a $(n+1)$-set of important constraints on the potential parameters that vary for different $n$. These constraints together with (29) yield the exact solutions of the systems. It can be easily shown that the expansion coefficients $p_{m}$ 's in (27) satisfy the three-term recursion relation:

$$
p_{m+1}=\frac{\left(2 b_{2}\right) p_{m-1}-\left(c_{0}+m\left(b_{1}+m-1\right)\right) p_{m}}{(m+1)\left(b_{0}-m a\right)}
$$

with the boundary conditions $p_{n+1}=0$ and $p_{-1}=0$. Therefore, we have succeeded in obtaining the exact expressions for the energies, wavefunctions, and the allowed values of the potential parameters for the $n+1$ first states algebraically. The main advantage of our algebraic method is that we can quickly obtain the general solutions of the systems for any arbitrary $n$ from (27), (29), and (31) without the cumbersome numerical and analytical procedures usually involved in obtaining the solutions for higher states. In the following, we 
apply the above results to obtain explicit solutions for each of the four systems.

4.1. Nonpolynomial Potential. In this case, from (11) with (25), we get

$$
\begin{aligned}
& a=1, \\
& b_{2}=-\frac{2}{\beta \sqrt{2}}, \\
& b_{1}=\frac{2}{\beta \sqrt{2}}+l+\frac{7}{2}, \\
& b_{0}=-\left(l+\frac{3}{2}\right), \\
& c_{1}=-\frac{E}{2 \beta}+\frac{\alpha}{2 \beta^{2}}-\frac{1}{\beta \sqrt{2}}\left(l+\frac{7}{2}\right), \\
& c_{0}=\frac{E}{2 \beta}+\frac{l}{2}+1+\frac{1}{\beta \sqrt{2}}\left(l+\frac{3}{2}\right) .
\end{aligned}
$$

Then by (29), the closed form of the energy of the system is obtained as

$$
E_{n}=\frac{\alpha}{\beta}-\sqrt{2}\left(2 n+l+\frac{7}{2}\right)
$$

which together with (31) yields the exact solutions of the system. Also, from (10) and (27), the wavefunction of the model is obtained as

$$
\psi_{n}(z)=(1-z) z^{(l+1) / 2} e^{-z / \beta \sqrt{2}} \sum_{m=0}^{n} p_{m} z^{m}
$$

$$
n=0,1,2, \ldots \text {, }
$$

where the expansion coefficients $p_{m}$ 's satisfy the recursion relation:

$$
p_{m+1}=\frac{(-4 / \beta \sqrt{2}) p_{m-1}-(E / 2 \beta+l / 2+1+(1 / \beta \sqrt{2})(l+3 / 2)+m(2 / \beta \sqrt{2}+l+7 / 2+m-1)) p_{m}}{-(m+1)((l+3 / 2)+m)} .
$$

The results obtained for the first three states of this model are displayed in Table 1.

4.2. Screened Coulomb Potential. In this case, comparing (15) with (25), we obtain

$$
\begin{aligned}
& a=-\kappa \\
& b_{2}=-2 \sqrt{-2 E} \\
& b_{1}=2(-\sqrt{-2 E} \kappa+l+2), \\
& b_{0}=(2 l+4) \kappa \\
& c_{1}=2(-\delta-\gamma-\sqrt{-2 E}(l+2)),
\end{aligned}
$$

$$
c_{0}=-2(\kappa(l+1) \sqrt{-2 E}+\kappa \gamma-l-1)
$$

Then by (29) and (14), we obtain the following relations for the energy eigenvalues and the corresponding wavefunctions:

$$
\begin{aligned}
E_{n} & =\frac{-1}{2}\left(\frac{\delta+\gamma}{n+l+2}\right)^{2}, \\
\psi_{n}(r) & =(r+\kappa) r^{l+1} e^{-\sqrt{-2 E}(r+\kappa)} \sum_{m=0}^{n} p_{m} r^{m}, \\
n & =0,1,2, \ldots,
\end{aligned}
$$

which together with the determinant relation (31) give the exact solutions of this system. Also, the expansion coefficients $p_{m}$ 's obey the three-term recursion relation:

$$
p_{m+1}=\frac{(-4 \sqrt{-2 E}) p_{m-1}-(-2(\kappa(l+1) \sqrt{-2 E}+\kappa \gamma-l-1)+m(2(-\sqrt{-2 E} \kappa+l+2)+m-1)) p_{m}}{(m+1)(2 l+4+m) \kappa}
$$

The results for the ground, first, and second excited states of this model are reported in Table 2.

4.3. Singular Integer Power Potential. In this case, from (19) and (25), we have

$$
\begin{aligned}
& a=0 \\
& b_{2}=-2 \sqrt{-2 E} \\
& b_{1}=2\left(1+\frac{\xi}{\sqrt{2 \tau}}\right),
\end{aligned}
$$




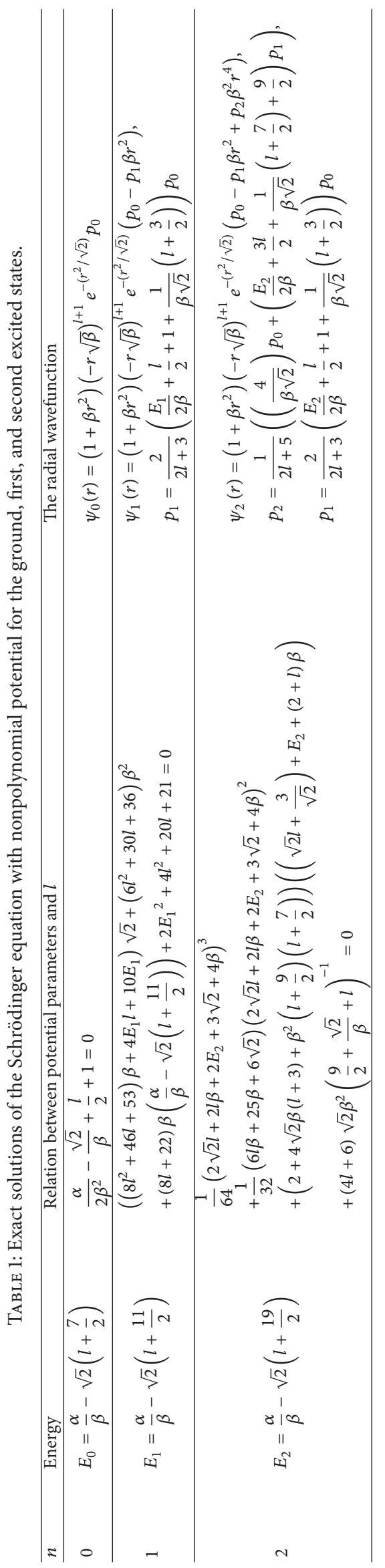




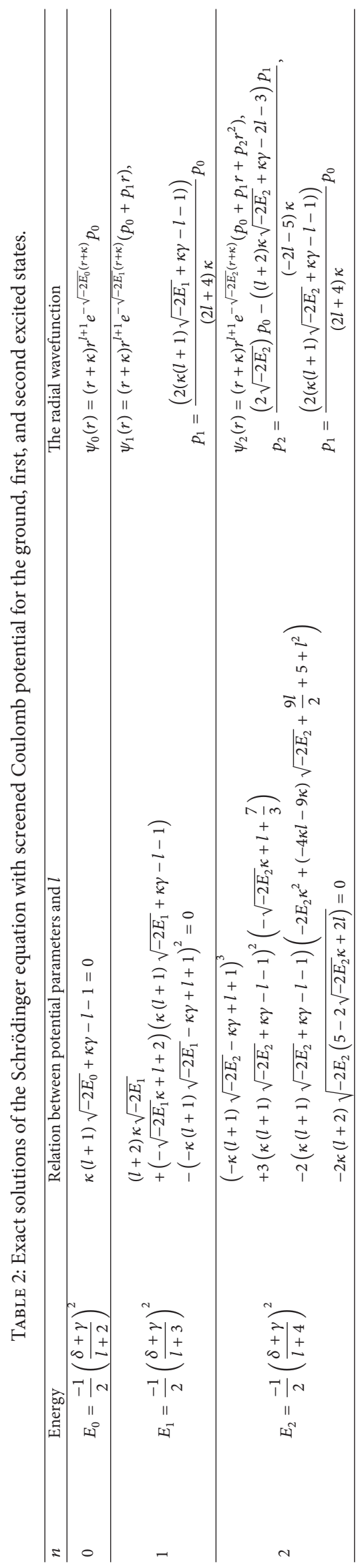




$$
\begin{aligned}
& b_{0}=2 \sqrt{2 \tau}, \\
& c_{1}=-2\left(\sqrt{-2 E}\left(1+\frac{\xi}{\sqrt{2 \tau}}\right)+\lambda\right), \\
& c_{0}=-2 \mu-l(l+1)-2 \sqrt{-4 \tau E}+\frac{\xi}{\sqrt{2 \tau}}+\frac{\xi^{2}}{2 \tau} .
\end{aligned}
$$

Then from (29) and (18), we obtain the following relations for energy and wavefunction:

$$
\begin{aligned}
E_{n} & =\frac{-\lambda^{2}}{2}\left(n+\frac{\xi}{\sqrt{2 \tau}}+1\right)^{-2}, \\
\psi_{n}(r)=r^{1+\xi / \sqrt{2 \tau}} e^{-(\sqrt{-2 E} r+\sqrt{2 \tau} / r)} \sum_{m=0}^{n} p_{m} r^{m} & \\
n & =0,1,2, \ldots,
\end{aligned}
$$

where the expansion coefficients $p_{m}$ 's satisfy the following recursion relation:

$$
p_{m+1}=\frac{(-4 \sqrt{-2 E}) p_{m-1}-\left(-2 \mu-l(l+1)-2 \sqrt{-4 \tau E}+\xi / \sqrt{2 \tau}+\xi^{2} / 2 \tau+m(2(1+\xi / \sqrt{2 \tau})+m-1)\right) p_{m}}{2(m+1) \sqrt{2 \tau}}
$$

These relations together with (31) yield the exact solutions of the system. The results determined for the ground, first, and second excited states are displayed in Table 3.

4.4. Singular Anharmonic Potential. In this case, from (23) and (25), we get

$$
\begin{aligned}
& a=0 \\
& b_{2}=-\sqrt{2 \omega} \\
& b_{1}=\left(2+\frac{\sigma}{\sqrt{2 \chi}}\right), \\
& b_{0}=\sqrt{2 \chi}, \\
& c_{1}=\frac{E}{2}-\sqrt{\frac{\omega}{2}}\left(2+\frac{\sigma}{\sqrt{2 \chi}}\right),
\end{aligned}
$$$$
p_{m+1}=\frac{(-2 \sqrt{2 \omega}) p_{m-1}+\left((1 / 4)\left(l^{\prime}\left(l^{\prime}+1\right)+2 \sqrt{4 \omega \chi}-\sigma^{2} / 2 \chi-2 \sigma / \sqrt{2 \chi}-3 / 4\right)-m((2+\sigma / \sqrt{2 \chi})+m-1)\right) p_{m}}{(m+1) \sqrt{2 \chi}}
$$

$$
c_{0}=-\frac{1}{4}\left(l^{\prime}\left(l^{\prime}+1\right)+2 \sqrt{4 \omega \chi}-\frac{\sigma^{2}}{2 \chi}-\frac{2 \sigma}{\sqrt{2 \chi}}-\frac{3}{4}\right)
$$

Then from (29) and (22), we obtain

$$
\begin{aligned}
& E_{n}=\sqrt{2 \omega}\left(2 n+\frac{\sigma}{\sqrt{2 \chi}}+2\right), \\
& \psi(r)=r^{3 / 2+\sigma / \sqrt{2 \chi}} e^{-\left(\sqrt{\omega / 2} r^{2}+\sqrt{\chi / 2}\left(1 / r^{2}\right)\right)} \sum_{m=0}^{n} p_{m} r^{m}, \\
& n=0,1,2, \ldots,
\end{aligned}
$$

for the energy and wavefunction, respectively, which together with (31) yield the exact solutions of the system. Also, the coefficients $p_{m}$ 's satisfy the recursion relation:
Solutions of the ground, first, and second excited states of this system are reported in Table 4.

\section{Conclusions}

In this paper, we have studied the Schrödinger equation for a class of spherically symmetric potentials and illustrated that these models can be treated in a simple and unified manner in the Lie algebraic approach. We have shown that all these models give rise to the same basic differential equation, which is expressible as an element of the universal enveloping algebra of $\mathrm{sl}(2)$. We have then obtained the general exact solutions of the basic equation within the framework of representation theory of $\mathrm{sl}(2)$ Lie algebra. Also, we have reported the explicit expressions for the energy, wavefunction, and the constraint on the potential parameters for each of the systems. The advantage of our algebraic method is that we can quickly obtain the general solutions of the systems for any arbitrary $n$, without the cumbersome procedures of obtaining the solutions for higher states. This method is found to be computationally much simpler than other methods.

\section{Competing Interests}

The authors declare that they have no competing interests. 


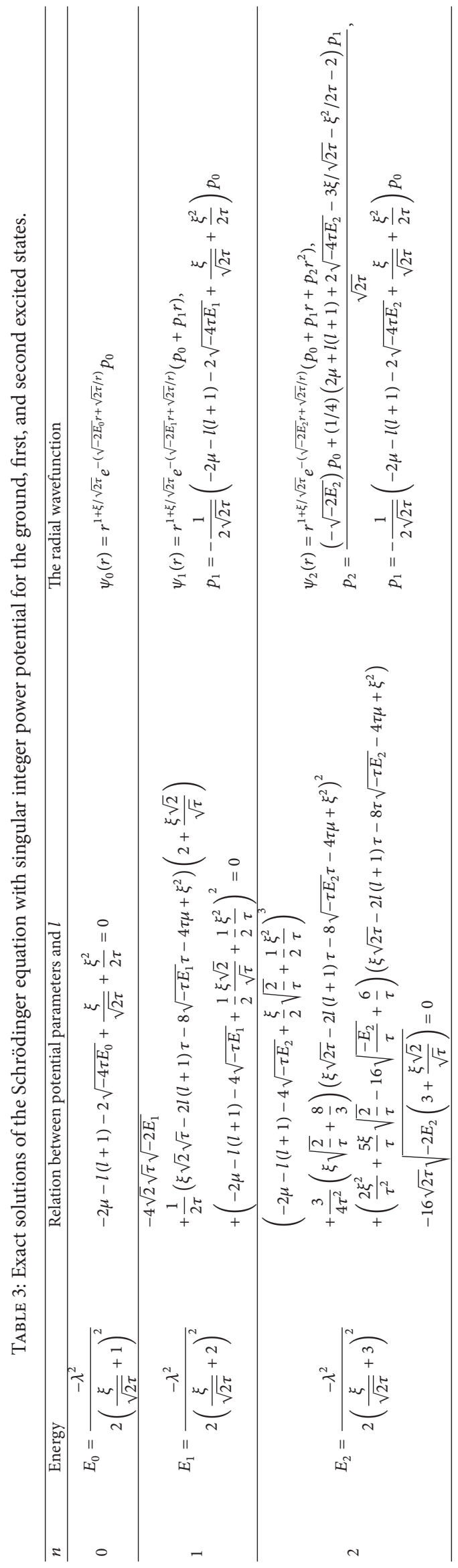




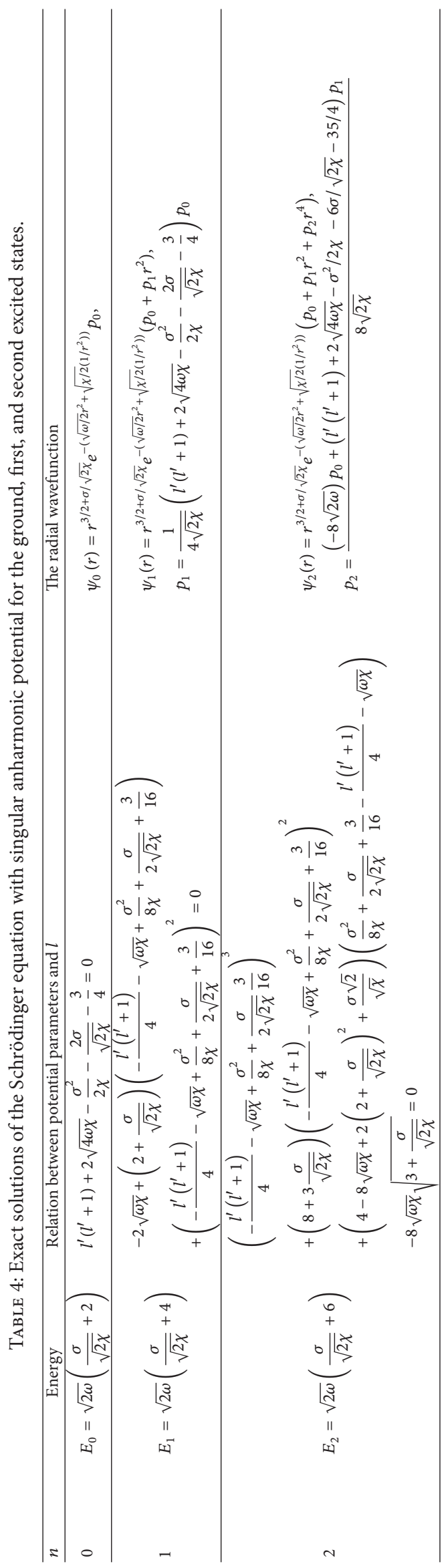




\section{References}

[1] G. Lèvai, "On some exactly solvable potentials derived from supersymmetric quantum mechanics," Journal of Physics A: Mathematical and General, vol. 25, no. 9, pp. L521-L524, 1992.

[2] F. Cooper, A. Khare, and U. Sukhatme, "Supersymmetry and quantum mechanics," Physics Reports, vol. 251, no. 5-6, pp. 267$385,1995$.

[3] S.-H. Dong, Factorization Method in Quantum Mechanics, vol. 150 of Fundamental Theories of Physics, Springer, Dordrecht, The Netherlands, 2007.

[4] M. Azizi, N. Salehi, and A. A. Rajabi, "Exact solution of the dirac equation for the yukawa potential with scalar and vector potentials and tensor interaction," ISRN High Energy Physics, vol. 2013, Article ID 310392, 6 pages, 2013.

[5] H. Panahi and L. Jahangiri, "Exact solution of the curved Dirac equation in polar coordinates: master function approach," Advances in High Energy Physics, vol. 2015, Article ID 612757, 8 pages, 2015.

[6] L. D. Landau and E. M. Lifshitz, Quantum Mechanics-Nonrelativistic Theory, Pergamon, New York, NY, USA, 1977.

[7] L. N. Chang, D. Minic, N. Okamura, and T. Takeuchi, "Exact solution of the harmonic oscillator in arbitrary dimensions with minimal length uncertainty relations," Physical Review D, vol. 65, no. 12, Article ID 125027, 8 pages, 2002.

[8] G. Pöschl and E. Teller, "Bemerkungen zur Quantenmechanik des anharmonischen Oszillators," Zeitschrift für Physik, vol. 83, no. 3-4, pp. 143-151, 1933.

[9] M. M. Nieto, "Exact wave-function normalization constants for the $B_{0} \tanh z-U_{0} \cosh ^{-2} z$ and Pöschl-Teller potentials," Physical Review A, vol. 17, no. 4, pp. 1273-1283, 1978.

[10] D. Griffiths, Introduction to Quantum Mechanics, Pearson, Hoboken, NJ, USA, 1995.

[11] S. M. Al-Jaber, "Hydrogen atom in N dimensions," International Journal of Theoretical Physics, vol. 37, no. 4, pp. 1289-1298, 1998.

[12] S. H. Dong, Wave Equations in Higher Dimensions, Springer, New York, NY, USA, 2011.

[13] P. M. Morse, "Diatomic molecules according to the wave mechanics. II. Vibrational levels," Physical Review, vol. 34, no. 1, pp. 57-64, 1929.

[14] G. Chen, "The exact solutions of the Schrödinger equation with the Morse potential via Laplace transforms," Physics Letters A, vol. 326, no. 1-2, pp. 55-57, 2004.

[15] N. Rosen and P. M. Morse, "On the vibrations of polyatomic molecules," Physical Review, vol. 42, no. 2, pp. 210-217, 1932.

[16] M. F. Manning and N. Rosen, "A potential function for the vibrations of diatomic molecules," Physical Review, vol. 44, p. 953, 1933.

[17] S.-H. Dong and J. García-Ravelo, "Exact solutions of the s-wave Schrödinger equation with Manning-Rosen potential," Physica Scripta, vol. 75, no. 3, pp. 307-309, 2007.

[18] T. Tietz, "Potential-energy function for diatomic molecules," Journal of Chemical Physics, vol. 38, no. 12, pp. 3036-3037, 1963.

[19] C. Eckart, "The penetration of a potential barrier by electrons," Physical Review, vol. 35, no. 11, pp. 1303-1309, 1930.

[20] F. L. Scarf, "New soluble energy band problem," Physical Review, vol. 112, pp. 1137-1140, 1958.

[21] A. K. Bose, "A class of solvable potentials," Il Nuovo Cimento, vol. 32, no. 3, pp. 679-688, 1964.
[22] J. N. Ginocchio, "A class of exactly solvable potentials. I. Onedimensional Schrödinger equation," Annals of Physics, vol. 152, no. 1, pp. 203-219, 1984.

[23] M. A. Shifman, "New findings in quantum mechanics (partial algebraization of the spectral problem)," International Journal of Modern Physics A, vol. 4, no. 12, pp. 2897-2952, 1989.

[24] A. G. Ushveridze, Quasi-Exactly Solvable Models in Quantum Mechanics, IOP, Bristol, UK, 1994.

[25] A. V. Turbiner, "Lie-algebras and linear operators with invariant subspaces," AMS eBooks: Contemporary Mathematics, vol. 160, p. $263,1994$.

[26] A. González-López, N. Kamran, and P. J. Olver, "Normalizability of one-dimensional quasi-exactly solvable Schrödinger operators," Communications in Mathematical Physics, vol. 153, no. 1, pp. 117-146, 1993.

[27] S. N. Biswas, K. Datta, R. P. Saxena, P. K. Srivastava, and V. S. Varma, "Eigenvalues of $\lambda x^{2 m}$ anharmonic oscillators," Journal of Mathematical Physics, vol. 14, no. 9, pp. 1190-1195, 1973.

[28] A. Salam and J. Strathdee, "Momentum-space behavior of integrals in nonpolynomial lagrangian theories," Physical Review D, vol. 1, no. 12, pp. 3296-3312, 1970.

[29] A. K. Mitra, "On the interaction of the type $\lambda x^{2} /\left(1+g x^{2}\right)$," Journal of Mathematical Physics, vol. 19, article 2018, 1978.

[30] G. P. Flessas, "On the Schrödinger equation for the $\mathrm{x} 2+\lambda \times 2(1+$ gx2) interaction,” Physics Letters A, vol. 83, no. 3, pp. 121-122, 1981.

[31] H. Risken and H. D. Vollmer, "The influence of higher order contributions to the correlation function of the intensity fluctuation in a Laser near threshold," Zeitschrift für Physik, vol. 201, no. 3, pp. 323-330, 1967.

[32] H. Haken, Laser Theory, Springer, Berlin, Germany, 1970.

[33] M. Znojil, "Potential $\mathrm{r}^{2}+\lambda \mathrm{r}^{2} /\left(1+\mathrm{gr}^{2}\right)$ and the analytic continued fractions," Journal of Physics A: Mathematical and General, vol. 16, no. 2, p. 293, 1983.

[34] S. K. Bose and N. Varma, "Exact solution of the Schrödinger equation for the central nonpolynomial potential $V(r)=r^{2}+$ $\lambda r^{2} /\left(1+g r^{2}\right)$ in two and three dimensions," Physics Letters A, vol. 141, no. 3-4, pp. 141-146, 1989.

[35] B. Roy, R. Roychoudhury, and P. Roy, "Shifted 1/N expansion approach to the interaction $\mathrm{V}(\mathrm{r})=\mathrm{r}^{2}+\lambda \mathrm{r}^{2} /\left(1+\mathrm{gr}^{2}\right)$," Journal of Physics A: Mathematical and General, vol. 21, no. 7, p. 1579, 1988.

[36] D. Agboola and Y.-Z. Zhang, "Unified derivation of exact solutions for a class of quasi-exactly solvable models," Journal of Mathematical Physics, vol. 53, no. 4, 042101, 13 pages, 2012.

[37] M. Znojil, "Analytic green function and bound states for the screened coulomb potential $V(r)=F / r^{2}+G / r+H /\left(r+z^{2}\right)$," Physics Letters A, vol. 94, no. 3-4, pp. 120-124, 1983.

[38] Y.-Z. Zhang, "Exact polynomial solutions of second order differential equations and their applications," Journal of Physics A: Mathematical and Theoretical, vol. 45, no. 6, Article ID 065206, 20 pages, 2012.

[39] M. E. Portnoi and I. Galbraith, "Variable-phase method and Levinson's theorem in two dimensions: application to a screened Coulomb potential," Solid State Communications, vol. 103, no. 6, pp. 325-329, 1997.

[40] G. C. Maitland, M. Rigby, E. B. Smith, and W. A. Wakeham, Intermolecular Forces, Oxford University Press, Oxford, UK, 1987.

[41] R. J. LeRoy and W. Lam, "Near-dissociation expansions in the spectroscopic determination of diatom dissociation energies: 
method, and application to BeAr+," Chemical Physics Letters, vol. 71, no. 3, pp. 544-548, 1970.

[42] E. Vogt and G. H. Wannier, "Scattering of ions by polarization forces," Physical Review, vol. 95, no. 5, pp. 1190-1198, 1954.

[43] L. D. Laudau and E. M. Lifshitz, Quantum Mechanics, Pergamon, Oxford, UK, 1977.

[44] S. H. Dong, "Schrödinger equation with the potential $V(r)=$ $A \bar{r}^{4}+B \bar{r}^{3}+C \bar{r}^{2}+D \bar{r}^{-1}$, Physica Scripta, vol. 64 , no. 4 , pp. $273-$ 276, 2001.

[45] S.-H. Dong, Z.-Q. Ma, and G. Esposito, "Exact solutions of the Schrödinger equation with inverse-power potential," Foundations of Physics Letters, vol. 12, no. 5, pp. 465-474, 1999.

[46] S.-H. Dong, "The Ansatz method for analyzing Schrödinger's equation with three anharmonic potentials in $D$ dimensions," Foundations of Physics Letters, vol. 15, no. 4, pp. 385-395, 2002. 

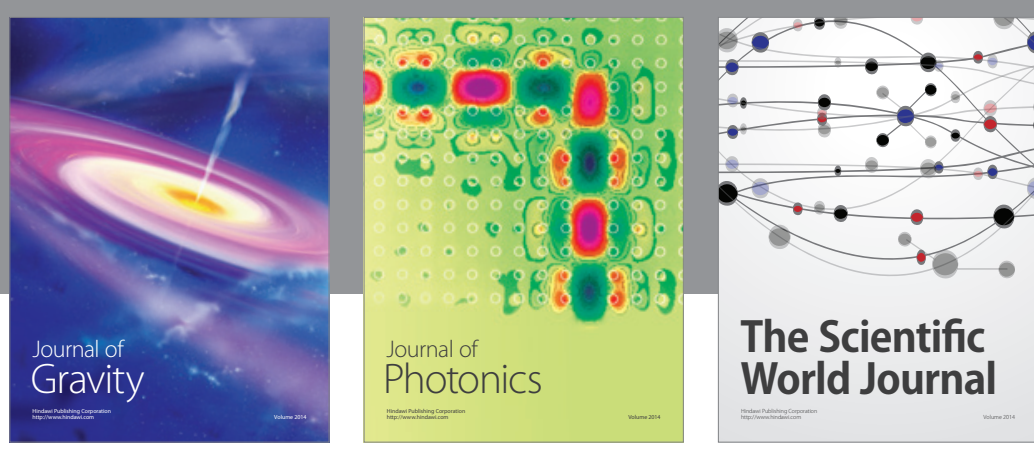

The Scientific World Journal
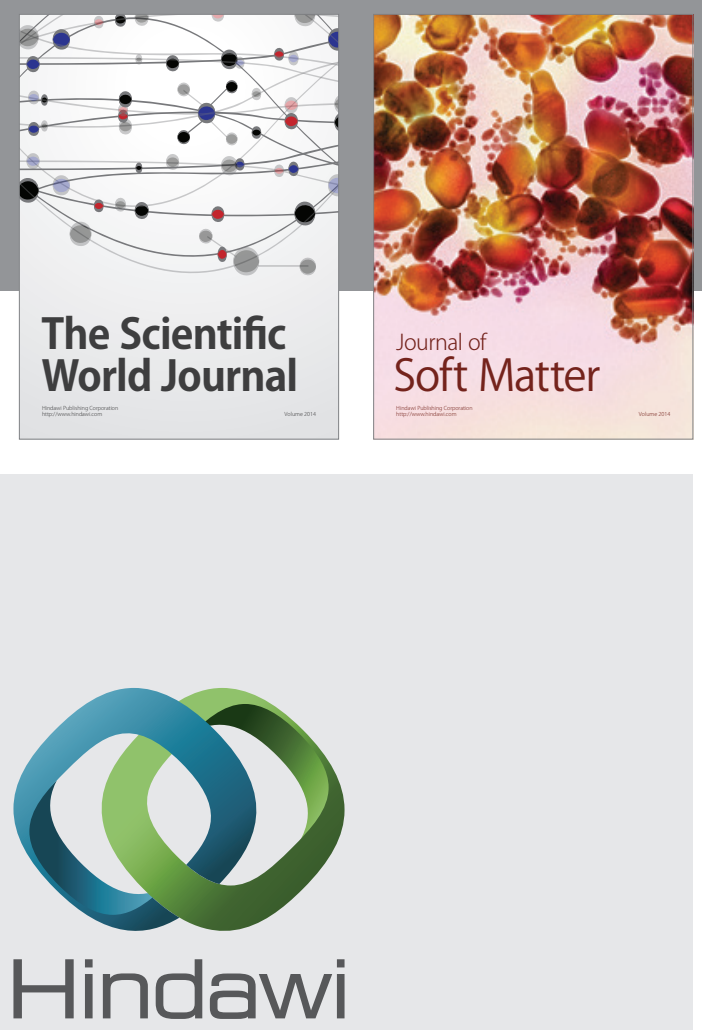

Submit your manuscripts at

http://www.hindawi.com

nternational Journal of

Statistical Mechanics
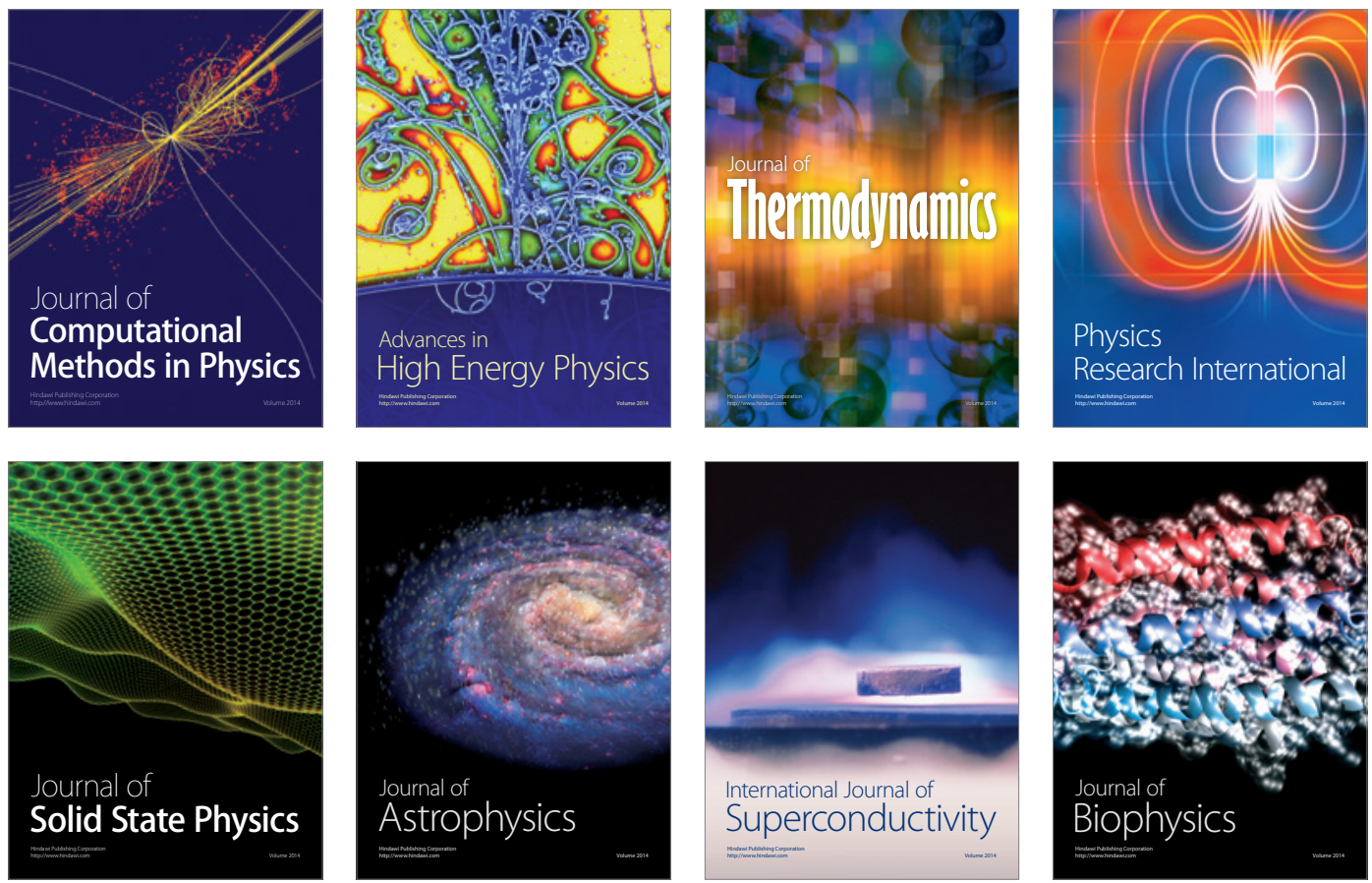
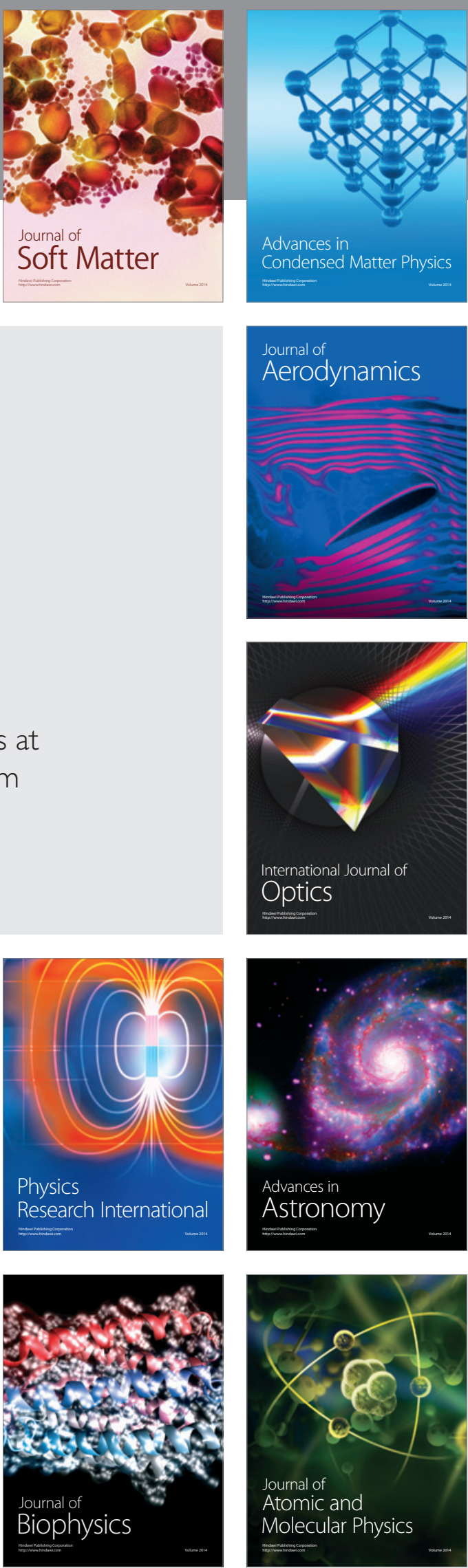